\title{
TRATAMENTO DA CANDIDÍASE VULVOVAGINAL E NOVAS PERSPECTIVAS TERAPÊUTICAS: UMA REVISÃO NARRATIVA
}

\section{TREATMENT OF VULVOVAGINAL CANDIDIASIS AND NEW THERAPEUTIC PERSPECTIVES: A NARRATIVE REVIEW}

\author{
Mariana Robatto Dantas Leal*, Maria Clara Neves Pavie Cardoso Lima**, Sibele de Oliveira \\ Tozetto Klein***, Patrícia Lordêlo****
}

Autora para correspondência: Patrícia Virginia Silva Lordelo Garboggi - pvslordelo@hotmail.com

*Fisioterapeuta, Mestre em Medicina e Saúde Humana, Doutoranda em Medicina e Saúde Humana pela Escola Bahiana de Medicina e Saúde Pública, pesquisadora do centro de Atenção ao Assoalho Pélvico, membro do grupo de pesquisa do CNPq Saúde da Mulher, Salvador, Bahia, Brasil

**Fisioterapeuta, Mestranda em Tecnologias em Saúde pela Escola Bahiana de Medicina e Saúde Pública, pesquisadora do Centro de Atenção ao Assoalho Pélvico, membro do grupo de pesquisa do CNPq Saúde da Mulher, Salvador, Bahia, Brasil

***Bióloga, Pós Doutora em Genética pela Universidade de São Paulo, professora colaboradora do Programa de Pós Graduação da Escola Bahiana de Medicina e Saúde Pública, professora adjunta do Centro de Ciências da Saúde, chefe do Núcleo de Intercâmbio da Superintendência de Assuntos Internacionais da Universidade Federal do Recôncavo da Bahia

****Fisioterapeuta, Pós Doutora em Ginecologia pela Universidade Federal de São Paulo, professora adjunta do Programa de Pós Graduação da Escola Bahiana de Medicina e Saúde Pública, coordenadora e fundadora do Centro de Atenção ao Assoalho Pélvico, coordenadora do grupo de pesquisa do CNPq Saúde da Mulher Salvador, Bahia, Brasil

\section{R E S U M O}

Introdução: A candidíase vulvovaginal (CVV) é um processo infeccioso do trato geniturinário inferior feminino causada pelo fungo Candida. No Brasil, é o segundo diagnóstico mais comum em ginecologia e tem ocasionado preocupação dos profissionais da saúde devido ao crescente número de casos recorrentes. A dificuldade na abordagem terapêutica da CVV aponta para a relevância do desenvolvimento de estudos que abordem as atuais terapias utilizadas para esta infecção e suas limitações, atentando os profissionais da área a desenvolver a visão crítica acerca do tema e buscar modificações no manejo clínico. Objetivo: Este estudo visa descrever o panorama atual dos tratamentos da CVV e as novas perspectivas terapêuticas. Estratégia Metodológica: Foi desenvolvida uma revisão de literatura contendo artigos em língua inglesa e portuguesa acerca dos atuais tratamentos existentes para CVV e possíveis perspectivas terapêuticas. Resultados: Nota-se que os casos de CVV, principalmente os recorrentes, têm apresentado limitação na resposta aos tratamentos medicamentosos atuais e por isto há uma crescente busca por inovações terapêuticas. Dentre as novas possibilidades, o Diodo Emissor de Luz (LED) azul 405 nm é uma possível modalidade de tratamento, no entanto, necessita-se de mais estudos que comprovem sua eficácia. Conclusão: A terapia antifúngica atual tem apresentado limitações, principalmente nos casos de recorrência e isto fortalece a necessidade de busca de novas modalidades terapêuticas que sejam capazes de tratar o processo infeccioso inibindo os mecanismos de resistência do microorganismo.

Palavras-chave: candidíase vulvovaginal; mulheres; tratamento; fototerapia. 
Introduction: The vulvovaginal candidiasis (VVC) is an infectious process of lower genitourinary tract and it's caused by Candida fungus. In Brazil, VVC is the second most common diagnosis and it has turned into a concern to health professionals because of its growing number of cases of recurrence. The difficulty in the therapeutic approach of VVC points to the importance of the development of studies that address the current therapies used for this infection and its limitations, considering the professionals to develop a critical view on the subject and seek changes in the clinical management. Objective: This study aims to describe the current panorama of the VVC treatment and its new therapeutic perspectives. Methodological Strategy: It was developed a literature review including article in portuguese and english language that discussed the issue of current treatments for VVC and possible new therapeutic. Results: It is noted that VVC cases, especially recurrent, have displayed some level of resistance to current drug treatment and because of that there is an increasing search for new types of therapeutic interventions. Among the new possibilities, the Light Emitting Diode (LED) in the blue color of $405 \mathrm{~nm}$ is a possible treatment mode however it's necessary more studies that can prove its effectiveness. Conclusion: The present antifungal therapy have shown limitations, mainly in cases of recurrence and this strengthens the need to search for new therapeutic modalities which are able to treat infectious processes by inhibiting microorganism resistance mechanisms.

Key words: vulvovaginal candidiasis, women, treatment, phototherapy. 


\section{INTRODUÇÃO}

A candidíase vulvovaginal (CVV) é um processo infeccioso do trato geniturinário inferior feminino, causada por várias espécies de cândida e que compromete principalmente a vulva e a vagina ${ }^{1,2}$. As principais espécies de interesse clínico são as subespécies de Candida albicans, glabrata, krusei, parapsilosis e tropicalis ${ }^{3}$.

A CVV é um relevante problema na saúde da mulher e um dos diagnósticos mais frequentes em ginecologia, sendo a segunda infecção genital mais comum no Brasil, precedida apenas pela vaginose bacteriana ${ }^{3,4,5}$. Estima-se que $75 \%$ das mulheres adultas apresentem pelo menos um episódio de vulvovaginite fúngica em sua vida, sendo que destas, $40 \%$ a $50 \%$ vivenciem novos surtos e $5 \%$ tornem-se recorrentes ${ }^{1,3,6,7}$ isto é, apresentam quatro ou mais episódios no período de um ano ${ }^{4}$.

Estudos demonstram que a Candida albicans é mais frequente que as espécies não-albicans, correspondendo a $80 \%$ dos casos. Porém, têm-se observado um aumento na frequência das espécies não albicans, especialmente Candida glabrata ${ }^{1,2,4,8}$. A maior preocupação reside no fato de que essas outras espécies tendem a ser mais resistentes aos antifúngicos ${ }^{1,9,10}$, considerado um importante fator de recorrência ${ }^{10}$.

A CVV caracteriza-se clinicamente pela ocorrência de prurido vulvar intenso, dispaurenia, disúria, edema e eritema vulvovaginal, sendo o prurido - sintoma mais importante quando comparado a vulvovaginites de outras etiologias ${ }^{2,3,9}$. Há também a presença de corrimento vaginal branco, espesso e inodoro ${ }^{2,5,9}$ e eventualmente podem surgir pontos branco-amarelados nas paredes vaginal e do colo uterino ${ }^{4,5}$.

O diagnóstico é estabelecido através da história e quadro clínico da paciente, porém deve ser confirmado por exames complementares, já que a sintomatologia não é patognomônica ${ }^{9,1}$.

O tratamento da CVV consiste no uso de agentes imidazólicos e triazólicos tópicos ou orais, além dos agentes poliênicos ${ }^{1}$, porém muitas mulheres não respondem à terapia inicial e os casos de recidivas são frequentes ${ }^{10}$, levando-as a iniciarem um tratamento cujo objetivo seja remissão clínica e microbiológica com consequente regime de manutenção ${ }^{6}$, que pode durar meses. Consequentemente, as pacientes podem desenvolver diversos efeitos adversos, como insônia, ansiedade, disúria e dor vaginal ${ }^{12}$.

Devido aos riscos associados ao uso de medicamentos e ao crescente índice de casos de recorrência, nota-se uma lacuna científica em relação a novas possibilidades terapêuticas viáveis. Terapias não medicamentosas vêm sendo desenvolvidas no tratamento desta patologia, já que são poucos os recursos disponíveis para tratar casos de resistência fúngica. Diante da importância epidemiológica e repercussões clínicas da CVV, o objetivo desta revisão é descrever os diferentes tratamentos para CVV, incluindo também os casos de recorrência, assim como, teorizar uma hipótese terapêutica inovadora com o uso do diodo emissor de luz (LED) fundamentando para futuros estudos clínicos.

\section{ESTRATÉGIA METODOLÓGICA}

Essa revisão foi realizada por meio de busca nas bases de dados Medline, Scielo e Lilacs, sendo utilizados os seguintes descritores: candidíase vulvovaginal, mulheres, tratamento e seus correlatos na língua inglesa: vulvovaginal candidiasis, women, treatment. Foram utilizados artigos nos idiomas português e inglês entre os anos de 2002 a 2014 . Após levantamento inicial, análise dos títulos, leitura dos resumos e exclusão das duplicatas, os artigos selecionados foram criticamente analisados.

Foram selecionados artigos de revisão, observacionais e experimentais que abordaram - tratamento da candidíase vulvovaginal e suas perspectivas terapêuticas. Foram excluídos editorais, resumos e estudos que abordaram 0 tratamento de vulvovaginites de outras etiologias e que envolvessem gestantes. 


\section{RESULTADOS/DISCUSSÃO}

\section{MÉTODOS DIAGNÓSTICOS}

diagnóstico baseia-se no quadro clínico, com o reconhecimento dos sinais e sintomas de prurido, corrimento esbranquiçado e espesso, com aspecto de "leite coalhado", hiperemia, edema e fissura em vulva e vagina. É necessária também a realização da microscopia a fresco com hidróxido de potássio (KOH) $10 \%$. Este exame deve ser associado à mensuração de $\mathrm{pH}$, que na CVV encontra-se normal $(\leq 4.5)$ e é realizado no próprio consultório, para detectar a presença de leveduras e/ou pseudohifas fúngicas, confirmando o diagnóstico ${ }^{1,8}$.

Nos casos recorrentes, deve-se acrescentar a realização de cultura vaginal específica, em meio de Sabouraud, realizada em laboratório, para especificar o subtipo de cândida envolvida no processo infeccioso ${ }^{1,8}$.

Sabe-se que na prática clínica, o diagnóstico da CVV é realizado baseado no quadro clínico apresentado, no entanto, nenhum dos sinais e sintomas são patognomônicos da patologia. Porém, devido ao elevado nível de incômodo relatado pela paciente, principalmente decorrente do prurido, muitas vezes - diagnóstico é realizado de forma empírica, levando a prescrição precipitada de medicamentos ou a automedicação. Esses fatores são vistos como os principais responsáveis pelo crescente índice de resistência fúngica medicamentosa, propiciando o crescimento dos casos de recorrência e dificultando o tratamento ${ }^{12}$.

\section{TRATAMENTO FARMACOLÓGICO}

O tratamento da CVV tem por objetivo, melhorar a sintomatologia da paciente, podendo ser efetuado por via oral ou tópica. No tratamento oral, a paciente pode se beneficiar de agentes imidazólicos e triazólicos: Fluconazol $150 \mathrm{mg}$ em dose única e Cetoconazol 200mg uma vez ao dia, por 14 dias $^{5}$. Já o tratamento tópico, deve ser via intravaginal com uso de Clotrimazol $1 \%$ durante 7 dias, Terconazol $0.8 \%$ aplicação de $5 \mathrm{~g}$ durante três dias e o ácido bórico $(600 \mathrm{mg} / \mathrm{supositório)} \mathrm{duas}$ vezes ao dia, por 14 dias5. Estes medicamentos alcançam cura clínica e microbiológica em $80 \%$ a $95 \%$ dos casos6. A terapia com agentes orais apresenta melhor resposta quando comparada aos antifúngicos tópicos?.

Para a manifestação da CVV recorrente, frequentemente é prescrito um tratamento antifúngico mais prolongado. No tratamento local, preconiza-se uso do clotrimazol (2 cápsulas de 100 $\mathrm{mg})$, duas vezes por semana durante seis meses ou uso tópico de terconazol $(0.8 \%)$ com aplicação de $5 \mathrm{~g}$ durante sete dias. $O$ tratamento oral engloba o uso de: cetoconazol ( 2 cápsulas de $200 \mathrm{mg}$ ) durante cinco dias após a menstruação, durante seis meses ou o uso de fluconazol (150 mg), administrados durante um mês ou ainda o uso de itraconazol $(200 \mathrm{mg}$ ) durante trinta dias ${ }^{5}$. Após esse período, a terapia é descontinuada. Caso haja recidiva, reinicia-se o tratamento, com regime de supressão, seguido de manutenção por um ano ${ }^{\circ}$.

Um estudo realizado por Moreira et al. (2004), comparou a ação de dois medicamentos, fluconazol (150 mg) e itraconazol (400 mg). As 181 pacientes receberam aleatoriamente um dos medicamentos e foram avaliadas do ponto de vista clínico e laboratorial em momentos distintos (7-21 dias e 2742 dias, respectivamente) e observou-se que houve redução dos sintomas, porém não houve diferença estatisticamente significante quando comparados $(p=0,247$ e $p=0,219)$, respectivamente. No entanto, nas pacientes que fizeram uso do itraconazol, a taxa de recidiva foi maior que nas que utilizaram - fluconazol $(p \leq 0,01)^{12}$. Este resultado pode ser explicado pelo fato de existir vantagem farmacocinética do fluconazol em relação ao itraconazol, pois as concentrações terapêuticas do primeiro no tecido vaginal permanecem por no mínimo três dias após a administração oral e no itraconazol esse tempo é ligeiramente menor, com duração máxima de três dias ${ }^{12}$.

Portanto, não há consenso sobre a superioridade de um medicamento em relação ao outro, devido à escassez de publicações sobre as atividades dos antifúngicos mais utilizados ${ }^{12}$. Porém, é relatada na literatura, a ocorrência de reações adversas com o uso de antifúngicos, principalmente naqueles de administração via oral, tais como: dor vaginal, infecção do trato urinário, insônia, ansiedade, desconforto gastrointestinal, disúria, dentre outros ${ }^{12}$. 


\section{TERAPIA FOTODINÂMICA}

A Terapia Fotodinâmica (TFD) é uma modalidade que vem sendo utilizada no tratamento de diversas patologias. Para sua realização, há necessidade de três elementos: droga fotossensibilizante, presença de oxigênio e uma fonte de luz, que pode ser o LASER (light amplification by stimulated emission of radiation) ou o LED (light emitting diode) ${ }^{13}$.

A luz é uma radiação eletromagnética cuja energia se transmite através de fótons ocupando o espectro de emissão, desde o ultravioleta, luz visível e infravermelho. A diferença importante entre o LASER e o LED é que a luz emergente do LED não é colimada nem coerente, o que representa uma limitação, já que a divergência do feixe de luz faz com que os fótons se repartam em uma superfície maior. Porém, essa característica apresenta uma vantagem, pois o terapeuta pode cobrir uma área maior, sem necessidade de movimentação do aplicador. O baixo custo e o fácil manuseio do LED, em relação ao LASER, aumentaram sua procura e utilização ${ }^{14}$.

Uma droga fotossensibilizante é capaz de absorver luz na presença de oxigênio e produzir espécies reativas de oxigênio, que reagem com componentes celulares dos microrganismos, gerando morte celular ${ }^{15}$. Dentre os fotossensibilizantes mais utilizados, pode-se citar o azul de metileno ${ }^{13}$.

Inúmeros estudos demonstram que alguns fotossensibilizantes podem ser ativados por luz e produzir efeito fungicida e bactericida, sem prejuízo para as células humanas ${ }^{15}$. No entanto, há carência de estudos na literatura sobre os efeitos da TFD em vaginites por Candida albicans. Entre os trabalhos existentes, a TFD é geralmente aplicada em modelos de candidíase oral ${ }^{16}$. Teichert et al. (2002) utilizaram laser associado ao azul de metileno na mucosa oral de murinos demonstrando redução da carga fúngica e maior efeito fungicida quando associado a maior concentração do fotossensiblizante ${ }^{17}$. Este resultado pode ser explicado pelo fato do azul de metileno, quando associado ao laser, apresentar elevada penetração na pele, com ação antimicrobiana contra fungos e bactérias ${ }^{17}$.

Junqueira et al. (2009) também trataram a candidíase bucal em ratos com laser e azul de metileno, porém os resultados não mostraram redução estatisticamente significante da carga fúngica, embora os ratos tratados apresentaram uma menor quantidade de lesões resultantes da candidíase do que os não tratados ${ }^{18}$. Diante deste achado, observa-se que a TFD pode ser uma alternativa promissora no tratamento das infecções fúngicas, mesmo não apresentando redução importante na quantidade de fungos, já que houve redução da inflamação e das lesões após 24 horas da aplicação da terapia ${ }^{18}$.

\section{FOTOTERAPIA COM USO DO LED AZUL 405 NM}

A fototerapia com uso do LED foi inicialmente utilizada em associação a substâncias fotossenbilizadoras, que constitui a TFD. No entanto, alguns estudos observaram que a luz utilizada de forma isolada e em comprimentos de onda menores, entre 400 e $410 \mathrm{~nm}$ (LED azul), inibiu o crescimento de inúmeros microrganismos, como fungos e bactérias, sendo que $\circ$ de $405 \mathrm{~nm}$ apresentou melhor resposta de inativação ${ }^{19,20}$.

Alguns estudos comprovam a ação fungicida do LED azul e seus efeitos variam dependendo da dose, comprimento de onda e natureza do microorganismo $0^{20,21,22}$. Pesquisas demonstram que a luz azul estimula a produção de citocinas próinflamatórias que incluem interleucinas 1 , fator de necrose tumoral e fator de estimulação de colônias de macrófagos e granulócitos ${ }^{23}$. Em processos inflamatórios, se a aplicação for imediata, além de acelerar a fisiologia da inflamação, reduz as lesões e consequentemente a dor ${ }^{24}$.

Além disso, na membrana plasmática e nas mitocôndrias dos fungos, há a presença de porfirinas, que são substâncias fotossensibilizadoras. Quando essas substâncias são expostas à luz azul, especificamente no comprimento de onda 405 nm, geram oxigênio singleto e radicais livres, que causam danos celulares irreversíveis, com consequente morte celular ${ }^{25}$. Este mecanismo de morte celular não é propício ao desenvolvimento de resistência microbiana $^{19}$.

Apesar da luz azul $405 \mathrm{~nm}$ ser germicida, ele encontra-se dentro de um espectro de comprimento de onda benigna e se for operado a níveis de irradiância adequados é seguro para exposição 
humana, tendo como reação adversa leve eritema na região tratada ${ }^{19}$. Inamura et al. (2014) realizaram um estudo cujo objetivo foi avaliar os efeitos da luz azul $405 \mathrm{~nm}$ em microrganismos associados à infecções persistentes da cavidade oral, dentre eles, a Candida albicans. Esta foi cultivada em colônias e dividida em três amostras, expostas a radiação da luz azul $405 \mathrm{~nm}$ com potência de 0,2 W e diferentes tempos de radiação e dose. Como resultados, verificaram que não houve efeito inibitório no tempo de 5 minutos (60J), porém nos tempos de 10 (120J) e 20 minutos (240J) houve uma redução de $60 \%$ e $90 \%$ na quantidade de fungos, respectivamente 20 . Estes resultados podem ser explicados pela presença de substâncias presentes no fungo, que atuam como fotossensibilizadores endógenos, sendo capazes de atrair e absorver a luz azul, gerando oxigênio atômico e radicais livres, com consequente morte celular ${ }^{20}$.

Ganz et al. (2005) realizaram um ensaio clínico com uso da luz azul $405 \mathrm{~nm}$ em região de mucosa gástrica de pacientes sintomáticos infectados cronicamente por $\mathrm{H}$. pylori para testar a hipótese de que esta luz poderia ser utilizada como método terapêutico para inativar e erradicar a bactéria. Como resultados, observou-se morte bacteriana significativa nas amostras tratadas quando comparadas com as amostras controle, com erradicação de pelo menos $90 \%$ das bactérias em sete dos nove pacientes. Além disso, não foram observadas diferenças histológicas entre as áreas tratada e não tratada, o que demonstra a segurança da metodologia utilizada ${ }^{26}$. Justificamse esses achados pela presença de porfirinas nas células bacterianas, que quando expostas à luz azul 405 nm, geram oxigênio singleto e outras espécies de oxigênio reativo, resultando na morte celular devido à ruptura de organelas e material cromossômico. Além disso, os parâmetros de dose e potência utilizados, apresentaram segurança, sem geração de calor e respeitando os limites para exposição do olho e pele à luz.

Portanto, sabendo que a CVV é uma patologia que tem alta prevalência entre mulheres em idade reprodutiva, que muitas não respondem à terapia inicial medicamentosa, tornando-se recorrentes, que $\circ$ LED apresenta baixo custo e fácil manuseio, além da necessidade da obtenção de tratamentos alternativos não invasivos e com um mínimo de reações adversas, faz-se necessária a elaboração de novos estudos, para avaliação dos efeitos do LED azul $405 \mathrm{~nm}$ no tratamento da CVV, como alternativa prática de tratamento para as mulheres cometidas com esta patologia.

\section{CONCLUSÃO}

Conclui-se que a terapia antifúngica medicamentosa utilizada no tratamento da CVV é eficaz, porém nos casos reincidentes, ela é falha, necessitando de mais pesquisas para desenvolvimento de novos recursos. O LED azul $405 \mathrm{~nm}$ parece ser uma alternativa de tratamento promissora nos casos de CVV recorrente, porém necessita ainda de estudos que comprovem a sua real eficácia.

\section{REFERÊNCIAS}

1. Ferrazza MHSH, Maluf MLF, Consolaro MEL, Shinobu CS, Svidzinski TIE, Batista MR. Caracterização das leveduras isoladas da vagina e sua associação com candidíase vulvovaginal em duas cidades do sul do Brasil. Rev Bras Ginecol Obstet. 2005;27(2):58-63. doi: 10.1590/S010072032005000200003

2. Holanda $A A R$, Fernandes $A C S$, Bezerra $C M$, Ferreira MAF, Holanda MRR, Holanda JCP et al. Candidíase vulvovaginal: sintomatologia, fatores de risco e colonização anal concomitante. Rev Bras Ginecol Obstet. 2007;29(1):3-9. doi: 10.1590/ S0100-72032007000100002

3. Camargo FP, Alves IA, Parlow MS, Goulart LS. Isolamento de cândida $s p$ da mucosa vaginal de mulheres atendidas em um serviço de ginecologia do município de Santo Ângelo-RS. NewsLab. 2008;87:96-104

4. Álvares CA, Svidzinsk TIE, Consolaro MEL. Candidíase vulvovaginal: fatores predisponentes do hospedeiro e virulência das leveduras. J Bras 
5. Peixoto JV, Rocha MG, Nascimento RTL, Moreira VV, Kashiwabara TGB. Candidíase: uma revisão de literatura. BJSCR. 2014;8(2):75-82

6. Feverschuette OTM, Silveira SK, Feverschuette I, Corrêa T, Grando L, Trepani A. Candidíase vaginal recorrente: manejo clínico. Femina. 2010;38(2):31 36

7. Galle LC, Gianinni MJSM. Prevalência e susceptibilidade de leveduras vaginais. J Bras Patol Med Lab. 2004;40(4):229-36. doi: 10.1590/ S1676-24442004000400004

8. Rosa MI, Rumel D. Fatores associados à candidíase vulvovaginal: estudo exploratório. RBGO. 2004;26(1):65-70. doi: 10.1590/S010072032004000100010

9. Ferracin I, Oliveira RMW. Corrimento vaginal: causa, diagnóstico e tratamento farmacológico. Infarma. $2005 ; 17(5 / 6): 82-6$

10. Rodrigues MT, Gonçalves AC, Alvim MCT, Filho DSC, Zimmermmann JB, Silva VL et al. Associação entre cultura de secreção vaginal, características sociodemográficas e manifestações clínicas de pacientes com diagnóstico de candidíase vulvovaginal. Rev Bras Ginecol Obstet. 2013;35(12):554-61. doi: 10.1590/S010072032013001200005

\section{Andrioli JL, Oliveira GSA, Barreto CS, Souza} $\mathrm{ZL}$, Oliveira $\mathrm{MCH}$, Cazorla IM et al. Frequência de leveduras em fluido vaginal de mulheres com e sem suspeita clínica de candidíase vulvovaginal. Rev Bras Ginecol Obstet. 2009;31 (6):300-4. doi: $10.1590 /$ S0100-72032009000600006

\section{Carvalho NS, Baracat EC, Naud PSV, Giraldo} PC, Simões JÁ, Duarte $G$ et al. Estudo multicêntrico comparativo entre fluconazol e itraconazol no tratamento da candidíase vulvovaginal. Rev Bras Med. 2002;59(4):244-9

\section{Donnelly RF, McCarron PA, Tunney MM, David} Woolfson A. Potential of photodynamic therapy in treatment of fungal infections of the mouth. Design and characterization of a mucoadhesive patch containing toluidine blue $O$. J Photochem Photobiol B. 2007;86(1):59-69. doi: 10.1016/i. iphotobiol.2006.07.011

14. Meyer PF, Araújo HG, Carvalho MGF, Tatum BIS, Fernandes ICAG, Ronzio OA et al. Avaliação dos efeitos do LED na cicatrização de feridas cutâneas em ratos Wistar. Fis Bras. $2010 ; 11(6): 428-32$

15. Sena RMM. Efeitos da terapia fotodinâmina mediada por laser de emissão vermelha e azul de metileno em vaginite induzida por Candida albicans [Doutorado em Ciências na Área de Tecnologia Nuclear- Materiais] - Instituto de Pesquisas Nucleares da Universidade de São Paulo: São Paulo; 2013

16. Mima EGDO, Pavarina AC, Dovigo LN, Vergani CE, Costa CADS, Kurachi $C$ et al. Susceptibility of Candida albicans to photodynamic therapy in a murine model of oral candidosis. Oral Surgery, Oral Med Oral Pathol Oral Radiol Endodontology. 2010;109(3):392-401. doi: 10.1016/i. tripleo.2009.10.006

17. Teichert MC, Jones JW, Usacheva MN, Biel MA. Treatment of oral candidiasis with methylene blue-mediated photodynamic therapy in an immunodeficient murine model. Oral Surgery, Oral Med Oral Pathol Oral Radiol Endodontology. 2002;93(2):155-60. doi: 10.1067/ moe.2002.120051

18. Junqueira JC, Martins Jda S, Faria RL, Colombo $C E$, Jorge $A O$. Photodynamic therapy for the treatment of buccal candidiasis in rats. Lasers Med Sci. $2009 ; 24(6): 877-84$. doi: 10.1007/s10103009-0673-4

19. Maclean M, Mckenzie K, Anderson JG, Gettinby G, Macgregor SJ. $405 \mathrm{~nm}$ light technology for the inactivation of pathogens and its potential role for environmental disinfection and infections control. J Hosp Infect. 201 4;88(1):1-1 1. doi: 10.1016/i. jhin.2014.06.004

20. Inamura T, Tatehara S, Takebe $Y$, Tokuyama R, Ohshima T, Maeda $\mathrm{N}$ et al. Antibacterial and antifungal effect of $405 \mathrm{~nm}$ monochromatic laser on endodontopathogenic 
microorganisms. Int J Photoenergy. 2014:1-7. doi: $10.1155 / 2014 / 387215$

21. Imada K, Tanaka S, Ibaraki Y, Yoshimura K, Ito $S$. Antifungal effect of 405-nm light on botrytis cinerea. Lett Appl Microbiol. 2014;59(6):670-6. doi: 10.1111 /lam. 12330

22. Guffey JS, Wilborn J. In vitro bactericidal effects of 405-nm and 470-nm blue light.

Photomed Laser Surg. 2006;24(6):684-8. doi: $10.1089 /$ pho.2006.24.684

23. Goldberg DJ, Russell BA. Combination blue $(415 \mathrm{~nm})$ and red (633 nm) LED phototherapy in the treatment of mild to severe acne vulgaris. J Cosmet Laser Ther. 2006;8(2):71-75. doi: $10.1080 / 14764170600735912$

24. Vinck E. Applicability of light emitting diode irradiation in physiotherapy. Photomed Laser Surg. 2005;23(2):167-171

25. Murdock LE, Mckenzie K, Maclean M, Macgregor SJ, Anderson JG. Lethal effects of high-intensity violet 405 -nm light on saccharomyces cerevisiae, candida albicans and on dormant and germinating spores of aspergillus niger. Fungal Biol. 2013;1 17(7-8):519-27. doi: 10.1016/i. funbio. 2013.05 .004

26. Ganz RA, Viveiros J, Ahmad A, Ahmadi A, Khalil A, Tolkoff J et al. Helicobacter pylori in patients can be killed by visible light. Lasers Surg Med. 2005;36(4):260-265. doi: 10.1002/ Ism.20161 